Rev Chil Salud Pública 2012;

Vol 16 (2): $156-162$

Tema de actualidad

\title{
Aspectos socioculturales de las intervenciones en salud
}

\author{
SOCIOCULTURAL ASPECTS OF HEALTH INTERVENTIONS
}

\section{INTRODUCCIÓN}

Cuando hablamos de evaluar intervenciones en salud lo primero es definir qué entendemos por ello. Una primera aproximación podría ser que es una acción en salud deliberada, dirigida a un grupo cuyo propósito es promover y/o cambiar el estado de salud de los beneficiarios a los cuales va dirigida. No obstante la claridad y obviedad respecto de su propósito, sus resultados dan cuenta de que no siempre se logran los efectos esperados en la población objetivo. Considerando la inversión que implica su implementación, cabe -entonces- hacerse la pregunta: ¿Qué hace que las intervenciones carezcan de los resultados esperados? Una experiencia exitosa es aquella que demuestra algún cambio en la conducta objetivo. Si deseamos que los hipertensos disminuyan el consumo de sal, esperamos que cambien el hábito de consumo de sal de excesivo a adecuado. Para ello -siguiendo a Perry'- ha de asumirse que la salud implica un conjunto de dimensiones y no solo la prevención de una muerte prematura o la ausencia de enfermedad, "salud es un estado dinámico de completo bienestar físico, psicológico, espiritual y social". Esto involucra intervenciones que han de tomar en cuenta al sujeto integralmente y no solo en términos de un cambio específico en un factor de riesgo. Se ha mostrado que una de las falencias en las intervenciones mediante la implementación de programas es la no consideración de los factores contextuales y socioculturales que enmarcan los procesos de salud y la cotidianeidad de las personas.

Además, cuando son evaluadas las intervenciones, se realizan en un contexto que no necesariamente corresponde a las condiciones cotidianas en las cuales se desenvolverán. En consecuencia, estas pueden mostrarse exitosas en un estudio "ficticio", lejano a la realidad en la cual tomará lugar.

Un cambio de conducta de las personas (hacer ejercicio, disminuir el consumo de sal y azúcar, hacerse un examen regular del PAP, etc.) implica una comprensión diferente de qué es salud; un cambio, por tanto, de creencias, empoderamiento del sujeto, que conlleva darse cuenta y hacerse responsable de su propia salud, adoptando acciones que beneficien su calidad de vida. Empoderamiento en salud es definido por Shearer ${ }^{2-5}$ como un proceso relacional, que considera la habilidad de deliberadamente participar en la salud y en las decisiones respecto de su propio cuidado. Ello incluye esfuerzos por optimizar la salud desde la perspectiva de la persona, desde lo que entiende qué es "mejorar" la salud o alcanzar un mejor "nivel de salud". El empoderamiento en

CRISTINADI SILVESTRE ${ }^{(1)}$

${ }^{(1)}$ Centro de Epidemiología Clínica. Facultad de Medicina. Universidad de Chile. Independencia 1027. Santiago. Chile. cparadizo@yahoo.es salud enfatiza la participación deliberada en el proceso de cambiar uno mismo y el propio medio ambiente, reconociendo los patrones y comprometiendo los propios recursos para lograr bienestar.

Una de las intervenciones en salud reproductiva es el screening citológico del cérvix uterino (conocido como PAP), para el cual se ha demostrado que las mujeres, pese a conocer la necesidad de hacerlo, no cumplen con la regularidad y continuidad esperada desde la racionalidad de un programa de carácter 
preventivo. ¿Qué hacer, entonces, para que una intervención tan relevante y costo efectiva sea más exitosa?

Un intento por responder a una pregunta tan relevante hace pensar en dos líneas posibles:

1. Tomar en cuenta los factores socioculturales que intervienen en las decisiones de hacer uso y el acceso a los programas de salud en el contexto particular en que es baja la adherencia al examen.

2. Sistematizar hallazgos de estudios que documentan experiencias exitosas de la implementación del Programa.

En este artículo expondremos sucintamente ambas líneas que podrían contribuir a lograr intervenciones más exitosas.

\section{Antecedentes del cáncer cervicouterino}

El cáncer cervicouterino es el segundo más común en el mundo ${ }^{6}$, con sobre 500.000 nuevos casos diagnosticados y 273.500 muertes estimadas anualmente, representa aproximadamente el $10 \%$ de la incidencia y mortalidad global de cáncer entre las mujeres ${ }^{7}$. Cerca del $85 \%$ del cáncer cervicouterino ocurre en los países en desarrollo ${ }^{10}$. Latinoamérica y otras zonas geográficas presentan las más altas tasas de incidencia (40.3 en América Central; 35.8 en el Caribe y 30.9 en América del Sur) ${ }^{6}$. En 2002 más de 32.000 mujeres murieron en Latinoamérica y el Caribe por su causa, comparada con las 22.000 muertes debidas a embarazos y causas relacionadas con el nacimiento ${ }^{7}$; específicamente en Chile mueren 700 mujeres anualmente 9 . Se estima una incidencia de 30 por 100.000 mujeres con una tasa de mortalidad de 9,6 por $100.000^{10}$. El cáncer cervicouterino es la única enfermedad cuyo screening citológico (Papanicolaou $\left.^{11}\right)$ permite detectar etapa precoces; es decir, al tratar la neoplasia intraepitelial (NIE), se impide un cáncer futuro. En otras palabras, es un ejemplo de enfermedad para la cual las desigualdades en sus resultados pueden ser superadas, dado que es fácilmente detectable, los medios de su diagnóstico tienen bajo costo y el tratamiento es efectivo si la condición es detectada en etapas tempranas ${ }^{12,13}$.

El problema reside entonces en que se diagnostica cuando ya se ha desarrollado 7 . Con el fin de reducir las muertes atribuibles al cáncer cervicouterino, es necesario hacer más exitosa la intervención, conocer cómo es otorgado el servicio, estimular y desarrollar el empoderamiento en salud de parte de la mujer, conocer las diferencias en características socioculturales de las mujeres beneficiarias y el contexto sociocultural e institucional que enmarca el proceso de otorgamiento del examen. Las muertes por esta causa son una evidencia de que el programa no está funcionando de acuerdo a los propósitos del mismo.

\section{Factores contextuales y socioculturales que determinan el resultado de los programa/ intervenciones en salud}

De importancia fundamental es la interacción cliente-proveedor de salud en el contexto de la organización del cuidado de la salud. Dentro de la organización en que se otorga el cuidado existen normas y roles específicos para su otorgamiento; deberes y derechos, normas que limitan a veces el otorgamiento de un servicio adecuado y eficiente. Las expectativas de los roles mutuos, lo que la mujer espera del agente de salud, lo que "aprendió" a esperar dado que atribuye ciertas particulares obligaciones a su rol y, a la vez, lo que el agente de salud espera de ella en tanto usuaria o clienta receptora del servicio. A la vez existe un conjunto de reglas de cómo realizar las atenciones de salud definidas y establecidas desde la perspectiva de cómo debe otorgarse un servicio de salud, desde la "racionalidad" de los agentes de salud, sin considerar los factores contextuales referidos a los usuarios. Por ende, la intervención es implementada en un grupo sin tomar en consideración sus particulares características socioculturales. Es decir, la relación entre usuario del programa y otorgador del mismo está influida por los distintos marcos de referencia de cada actor de la relación. En ellos la "calidad del cuidado" y, particularmente, la consideración de los factores presentes, contextuales y de nivel de análisis micro y macro, han permitido comprender mejor las necesidades que tienen las mujeres en salud reproductiva y sexual. Simmons y Elias $^{14}$ han propuesto un modelo que expone las dimensiones tanto latentes como manifiestas de 
la relación entre programa y cliente que tienen que ser medidas. Aún cuando elaboraron el modelo para explicar las interacciones proveedor y cliente de programas de salud reproductiva, este es aplicable para el caso del programa del PAP toda vez que las mismas dimensiones nos permiten responder a la pregunta: ¿Qué ocurre cuando los usuarios se vinculan con el sistema de otorgamiento de los servicios? Cuestión que se complementa con los hallazgos que coinci- den en evidenciar el papel del modo cómo es otorgado el servicio, en el éxito y continuidad de la adherencia. Los autores establecen una diferencia entre variables determinantes y variables consecuentes. Entre las variables determinantes en el caso del PAP a considerar serían: las políticas referidas al programa del PAP y a la administración del programa en cada contexto en particular, el sistema político administrativo y la estructura social y económica.

\section{Factores contextuales}

\section{Características del programa y de los}

usuarios

\section{Interacción cliente-proveedor}

\section{Efectos del servicio}

Adaptado de Simmons y Elías (1994).

Cantidad de contactos

- cobertura

- frecuencia

- duración os agentes proveedores

- Motivación

- Densidad

- Disponibilidad y accesibilidad

- Supervisión/liderazgo

Sistema político administrativo

Estructura social y económica
Logística

Características del cliente

- edad, número de hijos, estatus marital.

- alfabetismo

- roles de género

- estrato social

- experiencia con

servicio

reencias acerca de la salud
Servicios efectivamente otorgados/ planificados sancionados, no sancionados
- Satisfacción usuaria - Continuidad/Uso regular del programa - Logro de Metas del Programa del PAP
- relaciones interpersonales

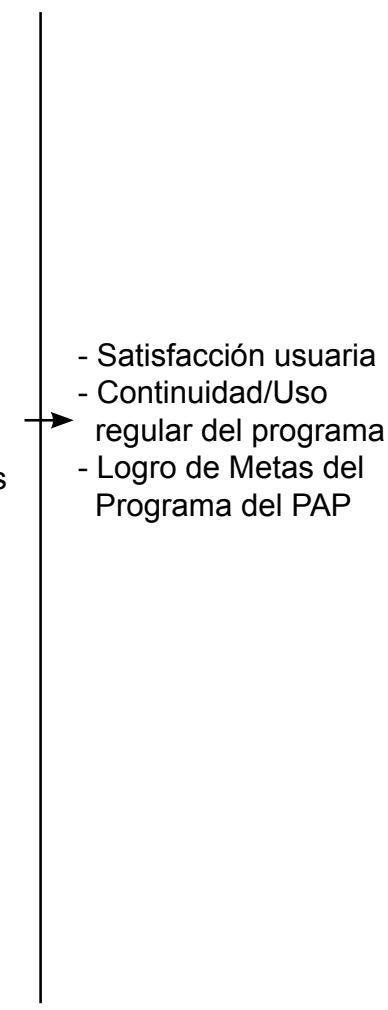

Figura 1. Interacciones entre cliente y proveedor del programa, sus determinantes y efectos del servicio. 
Estos factores contextuales en un nivel macrosocial estarían influyendo en el Programa y en las características del usuario: características de los proveedores, disponibilidad y accesibilidad de estos, motivación por el trabajo, lugar en que se otorga la intervención (Programa), supervisión (liderazgo) y logística, edad de la mujer, nivel educacional, roles de género, clase social de pertenencia, previas experiencias con el servicio otorgado y las creencias acerca de la salud. A su vez, ambos aspectos (características del programa y características de las mujeres/usuarias) están determinando, a nivel microsocial, las interacciones entre proveedor del servicio y cliente: a). Cantidad del contacto, cobertura, frecuencia y duración. b). Calidad de los contactos: elección, aspectos técnicos, relaciones interpersonales. Este conjunto de factores determinan la satisfacción usuaria, el hacerse el PAP, la regularidad en hacerse el examen y el logro de las metas del Programa. Este modelo podría ser aplicado en distintos contextos en que se otorga el programa a fin de evaluar las dimensiones determinantes y consecuentes del proceso para detectar los factores que están facilitando como aquellos que están limitando un otorgamiento exitoso de la intervención.

\section{Algunos hallazgos de estudios que documentan experiencias exitosas de la implementación del Programa}

Las experiencias exitosas han puesto en evidencia que la calidad del cuidado, la calidad de la relación entre usuario y sujeto y la satisfacción con el servicio otorgado ${ }^{15}$ son variables relevantes en el logro de una adherencia al programa del PAP y también la satisfacción con los servicios otorgados en el centro médico.

Respecto de la calidad del servicio otorgado Nash et al. ${ }^{16}$ mostraron el hecho que el agente de salud haya sugerido el hacerse los exámenes o preguntar a las mujeres sobre las recomendaciones respecto que se examinaran, influye positivamente en la búsqueda del servicio.

Truong Donnelly ${ }^{17}$ constató que la diferencia de estatus entre el médico y la usuaria y el hecho que la mujer percibe al médico en una posición superior, es decir existiendo una relación jerárquica, está asociada a cómo es recibido el examen.
Maxwell A. et al. ${ }^{18}$ demostraron en Filipinas, que las mujeres asistentes a una sesión educativa eran las que presentaban más retención en el programa de screening y menos resistencia para participar en el mismo. Se comprobó también que la invitación a sesiones grupales por parte de personas cercanas (amigas, conocidas) y la entrega de información por medio de estos encuentros constituye una estrategia efectiva para reclutar a mujeres para el screening de cáncer. Específicamente la asistencia a esas sesiones informativas mostró ser un predictor de retención de las mujeres al programa. Por otro lado, Haggstrom et al ${ }^{19}$ mostraron que el disponer de un plan de salud con derivación a un especialista aumenta la probabilidad que las mujeres se hagan el PAP. Además, se ha puesto en evidencia que la elaboración de guías clínicas tiene poco éxito en la retención de las mujeres, mientras que la invitación personal entregada por un «portero» o alguien que juega el papel de «receptor» de la mujer, informa y explica la necesidad de cuidado y prevención, - se asocian a un aumento en asistencia a los screenings ${ }^{19}$.

Un estudio que abordó los significados de hacerse el PAP y los procesos involucrados en la decisión de someterse a control periódico ${ }^{20}$ mostró que la experiencia de alguien cercano 0 familiar con cáncer gatilla el interés de las mujeres en acercarse al consultorio para hacerse el examen. Esto prueba la importancia de los "otros significativos" y el "modelo explicativo" del lego, en las acciones de salud que adoptan las personas. Los "otros significativos" lo conforman todas aquellas personas importantes en la vida del sujeto, influyendo en su vida $y$, particularmente, en la toma de decisiones que adopta. Entre estos se encuentran sus familiares y amigos, el individuo comparte con ellos un particular "modelo explicativo" el cual se refiere a las construcciones y representaciones (definiciones, ideas, juicios y creencias) respecto de la etiología, el momento y modo de presentación, el proceso fisiopatológico implicado, la historia natural, la severidad y el adecuado tratamiento de las enfermedades. Este modelo explicativo, la mayoría de las veces no corresponde al modelo explicativo de la biomedicina, lo que implica necesariamente que en el encuentro entre 
agente de salud y mujer se confrontan, además de otros factores, dos modelos explicativos que son parte de cada subcultura de pertenencia (la de la mujer y la del agente o proveedor del programa/cuidado de la salud). Los modelos explicativos tienen un origen social, es decir emergen y se desarrollan en los contextos socioculturales vitales de los individuos y son aprendidos durante el proceso de socialización. Durante este proceso cada ser humano es enseñado acerca de las posibles causas de las enfermedades, las expectativas de rol respecto de los agentes de salud y las normas sociales que definen su conducta en la situación de enfermedad y de búsqueda de ayuda médica. El modelo explicativo sostenido por la mujer va a influir en la evaluación de los tratamientos recibidos, concretamente en el sentido, en el cómo y qué características asume la toma del examen del PAP.

Los agentes de salud están moldeados en sus quehaceres y encuentro con las mujeres (usuarios) no solo por su socialización durante la infancia (socialización primaria), sino también por el proceso de socialización secundaria al cual fueron expuestos durante sus años de formación profesional, en los contextos de las escuelas de la salud (nutrición, obstetricia, enfermería, medicina, etc.). En tanto, instituciones sociales en las escuelas de ciencias de la salud existen valores, creencias, normas y expectativas de rol particulares, que son aprendidos, explícita e implícitamente- por los estudiantes, que influyen su conducta durante el encuentro con los usuarios (as). La consulta clínica puede ser comprendida como un intercambio entre dos modelos explicativos.

Por lo expuesto, es necesario adoptar una perspectiva que tome en cuenta el contexto cultural y social de los individuos, si pretendemos lograr mayor efectividad de las intervenciones. Al respecto, Kleinman ${ }^{21,22}$ postula que las creencias de las personas acerca de la enfermedad, incluyendo las expectativas que tienen respecto del tratamiento, afectan las formas como los individuos piensan acerca de y reaccionan a la enfermedad, escogen entre y evalúan la efectividad de las prácticas de cuidado de la salud disponibles para ellos. Cultura podemos definir- la como un conjunto de guías o lineamientos, tanto implícitos como explícitos, los cuales son heredados por cada persona en tanto miembro de una particular sociedad. La cultura se transmite de generación en generación a través del lenguaje, los símbolos y los rituales y provee a todo grupo humano de cohesión y continuidad ${ }^{23}$. Por ende, los conceptos de salud y enfermedad que sostienen los individuos de diferentes culturas influyen en las decisiones que estos adoptan respecto al cuidado de su salud. Como también las conductas de búsqueda de ayuda en salud, son moldeadas por las creencias, valores y las prácticas de la cultura de pertenencia del sujeto.

Con la subcultura de pertenencia se relacionan las posiciones sociales que tienen los individuos en una particular sociedad. Particularmente las diferencias de clase social entre el otorgador del programa (médico, matrona, enfermera) y la usuaria, establecen también diferencias en valores y en las creencias respecto de la naturaleza y causalidad de las enfermedades. Podemos considerar que en la relación entre el otorgador del servicio y el beneficiario (mujer) se encuentran dos subculturas, lo cual implica que inevitablemente factores tales como la mutua sospecha, la poca claridad del agente de salud en la explicación otorgada y la falta de comprensión por parte de la mujer pueden interferir en una comunicación efectiva y en la adopción regular de un examen preventivo. Está ampliamente constatado que la naturaleza de la comunicación verbal entre el médico y el paciente está asociada a la satisfacción y respuesta al consejo médico, como también que en la medida que las expectativas respecto al cuidado de su salud, trato y el modo en que es otorgado el servicio se cumplan, el usuario (paciente/mujer) se encontrará satisfecho (a) y lo más relevante es que se adherirá al tratamiento ${ }^{24}$; es decir en nuestro caso, se hará regularmente el examen del PAP.

Es relevante que los proveedores del cuidado de la salud y del examen del PAP tomen en cuenta la cultura de la mujer usuaria del servicio $y$, por ende, del concepto del cuerpo como algo privado en ella, su status o nivel socioeconómi$\mathrm{co}$, la relación proveedor de salud y paciente y el probable bajo nivel soporte institucional, como 
variables que determinan la continuidad en el cuidado y la aceptación de las intervenciones.

En síntesis, considerando algunos de los hallazgos expuestos y la importancia de la naturaleza y factores presentes en el encuentro entre agente de salud y usuaria, postulamos que sería relevante realizar una evaluación de proceso en el Programa del PAP que actualmente, se está implementando en Chile a fin de lograr mayor adherencia y continuidad de parte de las mujeres. Una "evaluación de proceso" ${ }^{25}$ está focalizada en el "cómo" se está implementando el Programa más que en sus resultados. Con esta evaluación podremos obtener información de cómo los participantes (usuarias, agentes de salud) están percibiendo la intervención, cómo es la dinámica interna de la atención otorgada, de la organización que apoya este programa, cómo se desarrollan los encuentros y relaciones entre las usuarias y los proveedores, entre los proveedores y la organización a la que pertenecen, la importancia o no de este programa en esos particulares contextos, los supuestos implícitos (no evidentes) en que está basado el otorgamiento del mismo que tienen que ver con la subculturas y modelos explicativos de los participantes de los encuentros y del contexto sociocultural tanto de las usuarias como de los proveedores del servicio. ¿Qué modelos explicativos sostienen cada cual (proveedor y mujer)? ¿Qué experiencias sostienen las personas que hacen que el Programa sea lo que es en su implementación concreta? ¿Cuáles son sus debilidades y fortalezas? ¿Qué sentimientos y actitudes están presentes en estos encuentros? ¿Qué acuerdos y desacuerdos existen entre ambos modelos explicativos? ¿Cómo, estos desacuerdos, perjudican o limitan una intervención exitosa? La naturaleza de las preguntas que nos hacemos: preguntas de proceso, significados, percepciones y el interés por "descubrir" lo que no está explícito en la relación agente de salud y mujer. Preguntas cuyas respuestas pueden requerir un diseño de investigación cualitativo.

\section{REFERENCIAS}

1. Perry Ch. Creating Health Behavior Change. Sage Publications, California; 1999.

2. Shearer N.C. Toward a Nursing Theory of Health Empowerment in Homebound Older Women. Journal of Gerontological Nursing 2007; December: 38-45.
3. Shearer N.C. Relationship of Contextual and Relational Factors to Health Empowernent in Women. Research and Theory for Nursing Practice: An International Journal 2004; 18, Nro. 4:357-370.

4. Shearer N.C Hair, Hands, and Oxygen Tanks: Embodiment and Health Empowerment in Homebound Older Women. Visions, Vo.15 Number 1, 2008.

5. Shearer N.N. Enpowerment: Reformulation of a Non-Rogerian Concept. Nursing Science Quarterly 2004;17, Nro.3:253-259.

6. World Health Organization, Cervical Cancer Screening in Developing Countries, Report of a WHO Consultation, 2002.

7. Alliance for Cervical Cancer Prevention, The Case for Investing in Cervical Cancer Prevention. Seattle:ACCp; 2004. Cervical Cancer Prevention Issues in Depth, Nro.3.

8. UNDP/UNFPA/WHO/World Bank Special Programme of Research, Development and Research Training in Human Reproduction. Progress in Reproductive Health Research, Nro. 65, 2004.

9. Ministerio de Salud Los Objetivos Sanitarios para la Década 2000-2010 (Octubre 2002).

10. Suárez E., Prieto M., y cols. Programa Nacional de Cáncer Cervicouterino. Rev. Chil Obstet Ginecol, 2001; 66 (6): 480-491.

11. El Papanicolaou (PAP) es un test citológico que detecta células cervicales anormales.

12. Roberts Ch.W.I. Health Care Disparities and Cervical Cancer. American Journal of Public Health, Dec. 2004. Vol. 94 Issue 12, p 2098- XXXX

13. Organización Mundial de la Salud Progress in Reproductive Health Research, Nro. 65, 2004.

14. Simmons R. y Elías Ch. The Study of Client-Provider Interactions: A Review of Methodological Issues. Studies in Family Planning 1994, January/February 25 (1):

15. Winkler J, Bingham A, Coffey P, Handwerker P, Women's Participation in a Cervical Cancer Screening Program in Northern Peru. Health Education Research 2008; 23 (1):10-24.

16. Nash D, Chan Ch, Horowitz D, Vlahov D. Barriers and Missed Opportunities in Breast and Cervical Cancer Screening among Women Aged 50 and Over, New York City, 2002. Journal of Women's Health 2007; 16(1):46-56.

17. Truong Donnelly T. Challenges in Providing Breast and Cervical Cancer Screening Services to Vietnamese Canadian Women: the Healthcare Providers' Perspective. Nursing Inquiry 2008; 15(2): 158-168.

18. Maxwell A., Bastani R., Perlaminda V., y Warda U. Strategies to Recruit and Retain Older Filipino-American Inmigrants for a Cancer Screening Study, Journal of Community Health 2005; 30 (3):167-180.

19. Haggstrom D., Phillips K., Liang S., Haas J., Tye Sh., y Kerlikowske K. Variation in Screening Mammography and Papanicolaou Smear by Primary Care Physician Specialty and Gatekeeper Plan (United States). Can- 
cer Causes and Control 2004;15:883-892.

20. Radovcic J, González G, Lorenzetti L, Valdenegro M, Trayectorias de Mujeres con Papanicolaou Vigente, Universidad Diego Portales, Mayo, 1999.

21. Kleinman A. et al Culture, Illness, and Care. Clinical lessons from Anthropologic and Cross-Cultural Research. Annals of Internal Medicine 1978;88:251-258.

22. Kleinman A. The Meaning of Symptoms and Disorders. En Kleinman A, editor. The Illness Narratives: Suffering, Healing, and the Human Condition, New York: Basic Books; 1988. p. 213-45.
23. Helman C.G, Research in Primary Care: The Qualitative Approach. En Norton et al editors. Primary Care Research, Traditional and Innovative Approaches. Sage Publications, California, 1991. p.105-124.

24. Korsch B, Gozzi E, Francis V, Gaps in Doctor-Patient Communication. Pediatrics 1968;42 (5):855-871.

25. Patton M.Q, Particularly Appropriate Qualitative Applications. En Patton M.Q. Qualitative Evaluation and Research Methods. Second Edition. Sage Publications; 1990. p. 92-141.

Recibido: 14 de junio de 2011 Aprobado: 30 de mayo de 2012 